\title{
High prevalence of multidrug resistant Enterobacteriaceae isolated from outpatient urine samples but not the hospital environment in Bo, Sierra Leone
}

Tomasz A. Leski ${ }^{1 *}$, Chris R. Taitt ${ }^{1}$, Umaru Bangura ${ }^{2,4}$, Michael G. Stockelman¹, Rashid Ansumana 2,3,4, William H. Cooper $1 I^{5}$, David A. Stenger ${ }^{1}$ and Gary J. Vora ${ }^{1}$

\begin{abstract}
Background: The rising level of antimicrobial resistance among bacterial pathogens is one of the most significant public health problems globally. While the antibiotic resistance of clinically important bacteria is closely tracked in many developed countries, the types and levels of resistance and multidrug resistance (MDR) among pathogens currently circulating in most countries of sub-Saharan Africa are virtually unknown.
\end{abstract}

Methods: From December 2013 to April 2014, we collected 93 urine specimens from all outpatients showing symptoms of urinary tract infection (UTI) and 189 fomite swabs from a small hospital in Bo, Sierra Leone. Culture on chromogenic agar combined with biochemical and DNA sequence-based assays was used to detect and identify the bacterial isolates. Their antimicrobial susceptibilities were determined using a panel of 11 antibiotics or antibiotic combinations.

Results: The 70 Enterobacteriaceae urine isolates were identified as Citrobacter freundii $(n=22)$, Klebsiella pneumoniae $(n=15)$, Enterobacter cloacae $(n=15)$, Escherichia coli $(n=13)$, Enterobacter sp./Leclercia sp. $(n=4)$ and Escherichia hermannii $(n=1)$. Antimicrobial susceptibility testing demonstrated that $85.7 \%$ of these isolates were MDR while $64.3 \%$ produced an extended-spectrum ß-lactamase (ESBL). The most notable observations included widespread resistance to sulphonamides (91.4\%), chloramphenicol (72.9\%), gentamycin (72.9\%), ampicillin with sulbactam (51.4\%) and ciprofloxacin (47.1\%) with C. freundii exhibiting the highest and E. coli the lowest prevalence of multidrug resistance. The environmental cultures resulted in only five Enterobacteriaceae isolates out of 189 collected with lower overall antibiotic resistance.

Conclusions: The surprisingly high proportion of C. freundii found in urine of patients with suspected UTI supports earlier findings of the growing role of this pathogen in UTIs in low-resource countries. The isolates of all analyzed species showed worryingly high levels of resistance to both first- and second-line antibiotics as well as a high frequency of MDR and ESBL phenotypes, which likely resulted from the lack of consistent antibiotic stewardship policies in Sierra Leone. Analysis of hospital environmental isolates however suggested that fomites in this naturally ventilated hospital were not a major reservoir for Enterobacteriaceae or antibiotic resistance determinants.

Keywords: Citrobacter, MDR, ESBL, CHROMagar, Sierra Leone

\footnotetext{
* Correspondence: tomasz.leski@nrl.navy.mil

${ }^{1}$ Naval Research Laboratory, Code 69104555 Overlook Avenue SW,

Washington, DC 20375, USA

Full list of author information is available at the end of the article
} 


\section{Background}

The increasing rate of antibiotic resistance among bacterial pathogens causing both hospital- and community-acquired infections is a serious threat to public health worldwide [1, 2]. Trends among pathogens belonging to the Enterobacteriaceae are especially troubling considering their ubiquity in the environment and animal hosts and the relative ease with which they acquire and transfer genetic determinants that confer resistance to most classes of antibiotics. Recently, this phenomenon has resulted in the emergence and international spread of bacteria causing a number of deadly hospital outbreaks [3, 4]. These include increasingly abundant and problematic bacterial strains harboring extended-spectrum ß-lactamases (ESBL) that confer resistance to the majority of $ß$-lactam antibiotics and carbapenemases that confer resistance to carbapenem compounds, the most powerful and last line of $ß$-lactam antibiotics [5-9]. Typically, ESBL-producing strains, carbapenem resistant Enterobacteriaceae (CRE), and other multidrug resistant (MDR) Enterobacteriaceae possess several additional resistance mechanisms to other classes of popular antibiotics such as phenicols, sulfonamides, fluoroquinolones, tetracyclines, and aminoglycosides [10, 11]. This makes them extremely difficult - and in some cases, virtually impossible - to treat.

While MDR bacterial pathogens have been vigorously investigated in countries with mandated antibiotic stewardship policies and adequate resources, scant data are available from developing countries [12]. As a salient example, a recent survey of the antimicrobial susceptibility of Enterobacteriaceae that caused urinary tract infections (UTI) in Africa revealed that $<200$ studies were published between the years 2000 and 2012. Furthermore, although these analyses originated from 14 African countries, $90 \%$ of the analyzed isolates came from South Africa and only a handful of the studies were conducted in other sub-Saharan African countries [13]. This kind of data is critical for developing viable antibiotic use policies and therapeutic strategies, especially since these same antimicrobial compounds are available without prescription in many developing countries (often resulting in widespread misuse [14-16]) and the situation can be further confounded by the presence of counterfeit or substandard quality drugs $[17,18]$. To date, no official data on antimicrobial resistance of Enterobacteriaceae circulating in Sierra Leone are available [12]. To begin to gain some insight into the antibiotic sensitivity of Enterobacteriaceae in this area, we conducted a prospective study at Mercy Hospital (Bo, Sierra Leone) using bacterial isolates obtained from urine of patients with symptoms consistent with UTI and the samples collected from the surrounding hospital environment.

\section{Methods}

Bacterial isolates from clinical and hospital environmental samples

Mercy Hospital is a small private hospital in Bo, Sierra Leone, that refers about 2000 patients per year to its own clinical laboratory for testing [19]. Clinical samples for this study were obtained between December 12th 2013 and January 27th 2014 from all outpatients showing symptoms of UTI and referred for urine culture by the attending clinician. The samples originated from patients ranging in age from 1 to 59 with $63.4 \%$ of the patients being female.

A total of 93 urine samples were screened on CHROMagar Orientation medium (CHROMagar, Paris, France). Seventy isolates were obtained from samples originating from 59 patients with significant bacteriuria (some samples yielded more than one isolate due to mixed infections). They included 57 isolates producing colonies with a dark blue color after overnight incubation at $37{ }^{\circ} \mathrm{C}$ (a phenotype characteristic of Klebsiella, Enterobacter, Citrobacter and Serratia when grown on this medium). Although this study was designed with the overall goal to analyze the antimicrobial resistance patterns of the nonE. coli Enterobacteriaceae, given the accepted role of Escherichia coli as the most prevalent bacterial pathogen causing UTI, 13 isolates producing dark pink to reddish colonies (the E. coli phenotype on this medium) were also added to the study.

Environmental isolates were obtained from 189 swab samples collected in and around the hospital between January 12th, 2014 and April 29th, 2014. The swabbing was performed using sterile cotton swabs moistened with sterile saline. Surfaces of approximately $10 \mathrm{~cm}^{2}$ of the tested area were swabbed and the swab was then swept across a CHROMagar Orientation medium plate which was subsequently incubated overnight at $37{ }^{\circ} \mathrm{C}$. The areas swabbed included outpatient waiting areas, general and maternity wards, and waiting areas and benches in the diagnostic lab located in a separate building (Additional file 1: Table S1).

\section{Isolate identification}

All collected Enterobacteriaceae isolates were identified to the species level using the API 20E identification system (bioMérieux, Marcy-l'Etoile, France) and 16S rRNA and $r p o B$ gene sequencing. For the $16 \mathrm{~S}$ rRNA-based identification, a fragment of $16 \mathrm{~S}$ rDNA that spanned the V3 and V4 variable regions was PCR amplified and sequenced [20]. Genus- (or family-) level identification was determined using the naïve Bayesian classifier available through the Ribosomal Database Project (release 11) (http://rdp.cme.msu.edu/classifier/classifier.jsp), with $80 \%$ confidence as the identification threshold [21]. Identification based on $r p o B$ gene sequencing was 
performed using previously published primers [22, 23], with a BLAST search performed on the obtained amplicon DNA sequence. The highest-ranking BLAST result with the highest coverage was used as the basis for organism identification. A final consensus identification was based on a combination of the three identification methods (Table 1). In instances where the methods did not result in concordant identifications, a genus-level identification was assigned to the isolate based on the agreement of two of the methods. The species identification determined by $r р о B$ gene sequencing was used when the phenotypic identification method and rроB sequencing results were discordant. Clinical isolates for which API 20E-based identification was ambiguous (SL108, SL116, SL122, SL124, SL127) or for which the three identification methods gave discordant results (SL184, SL191, SL201 and SL204) were subjected to additional identification using the ID 32 rapid system (bioMérieux). Twenty-six of the non-Enterobacteriaceae environmental isolates were identified to the genus level only using $16 \mathrm{~S}$ rRNA gene sequencing.

\section{Antibiotic susceptibility testing and ESBL detection}

Every clinical and environmental isolate included in this study was assayed by disk diffusion for susceptibility to the following antibiotics or antibiotic combinations: ampicillin with sulbactam (SAM), aztreonam (ATM), ceftazidime (CAZ), chloramphenicol (C), ciprofloxacin (CIP), doxycycline (D), gentamicin (GM), imipenem (IMP), kanamycin (K), sulfisoxazole (G), and tigecycline (TGC). The susceptibility testing was conducted using BBL Mueller Hinton II Agar (MHA medium, Becton Dickinson, Franklin Lakes, NJ) according to Clinical and Laboratory Standards Institute (CLSI) recommendations [24]. CLSI interpretative criteria were applied for classification of the isolates into susceptible, intermediate and resistant categories [24]. In the case of tigecycline, for which there are no CLSI criteria, FDA recommended breakpoints were used [25]. Minimum inhibitory concentrations (MIC) for imipenem were determined using imipenem E-test strips (bioMérieux) according to manufacturer's recommendations. The ESBL phenotype was determined using cefotaxime/cefotaxime + clavulanic acid (CT/CTL) and ceftazidime/ceftazidime + clavulanic acid (TZ/TZL) E-test strips (bioMérieux) according to manufacturer's recommendations or by the disk diffusion method using disks containing cefotaxime/cefotaxime + clavulanic acid and ceftazidime/ceftazidime + clavulanic acid according to CLSI recommendations [24]. E. coli reference strain ATCC 25922, susceptible to all 11 antibiotics tested in this study, was used for quality control purposes. The MDR phenotype was defined as the demonstrated resistance to at least three antibiotics belonging to different classes of antimicrobial compounds.
PCR-based detection of $b l a_{\text {CTX-M }}$

All of the isolates were tested for the presence of genes encoding CTX-M type ESBLs using universal CTX-M primers as previously described [26].

\section{Results \\ Identification of isolates from urine cultures}

Out of the 93 urine samples tested, 57 generated dark (metallic) blue colonies after an overnight incubation on CHROMagar Orientation medium, which was indicative of non-E. coli Enterobacteriaceae. These isolates were subcultured on MHA medium and identified using a combination of biochemical and molecular techniques. The consensus identification results for the clinical Enterobacteriaceae isolates are presented in Table 1 and the complete identification results are available in Additional file 2: Table S2. The consensus identification results revealed that the largest number of non-E. coli Enterobacteriaceae isolates were Citrobacter freundii $(n=22)$, followed by Enterobacter cloacae and Klebsiella pneumoniae ( $n=15$ isolates each) and Escherichia hermannii $(n=1)$. We were not able to identify unequivocally the genus of four non-E. coli Enterobacteriaceae isolates (SL184, SL191, SL201 and SL204) due to divergent phenotype identification results and ambiguous results of $16 \mathrm{~S}$ rRNA and rpoB sequence based analysis. The sequencebased identification indicated that these four isolates belong to a non-E. cloacae species of Enterobacter or to Leclercia sp. (data not shown). This group of four isolates is therefore referred to as "Enterobacter sp./Leclercia sp." All 13 isolates producing dark pink to reddish colonies included in the study were confirmed as E. coli.

\section{Enterobacteriaceae in the hospital environment}

To explore the role of fomites as reservoirs or agents in the transmission of MDR Enterobacteriaceae, hospital surfaces were also examined via swab and culture. An initial set of 95 samples, collected between January 12th and 15th, 2014, resulted in 30 isolates producing dark blue colonies on CHROMagar Orientation medium. However, subsequent subculture, biochemical analyses, and $16 \mathrm{~S}$ rRNA gene sequencing revealed that only four of these 30 isolates belonged to the Enterobacteriaceae. The remaining isolates were identified as Bacillus sp. $(n=23)$ and Staphylococcus sp. $(n=3)$, Additional file 2: Table S2. The analysis of an additional set of 94 hospital environmental samples, collected between March 31st and April 29th, 2014, yielded only one additional Enterobacteriaceae isolate, totaling only five Enterobacteriaceae isolates among the 189 environmental samples analyzed (2.7\%). Species-level identifications of these five isolates revealed two $K$. pneumoniae, two $E$. cloacae and one Pantoea dispersa. 
Table 1 Antibiotic sensitivity testing results

\begin{tabular}{|c|c|c|c|c|c|c|c|c|c|c|c|c|c|c|c|}
\hline \multirow[t]{2}{*}{ ID } & \multirow[t]{2}{*}{ Identification $^{a}$} & \multicolumn{11}{|c|}{ Disk diffusion $^{b}$} & \multirow{2}{*}{$\begin{array}{l}\text { E-test } \\
\text { IMP }\end{array}$} & \multicolumn{2}{|l|}{$\mathrm{ESBL}^{\mathrm{C}}$} \\
\hline & & ATM & CAZ & CIP & IMP & TGC & C & D & G & GM & K & SAM & & Phenotype & CTX-M \\
\hline \multicolumn{16}{|c|}{ Clinical isolates } \\
\hline SL122 & Citrobacter freundii & $\mathrm{R}$ & $\mathrm{R}$ & $\mathrm{R}$ & S & S & $\mathrm{R}$ & $\mathrm{R}$ & $\mathrm{R}$ & $\mathrm{R}$ & $\mathrm{R}$ & R & 0.250 & YES & YES \\
\hline SL124 & & $\mathrm{R}$ & । & $\mathrm{R}$ & S & S & $\mathrm{R}$ & $\mathrm{R}$ & $\mathrm{R}$ & $\mathrm{R}$ & $\mathrm{R}$ & $\mathrm{R}$ & 0.380 & YES & YES \\
\hline SL127 & & $\mathrm{R}$ & I & $\mathrm{R}$ & $\mathrm{S}$ & 1 & $\mathrm{R}$ & $\mathrm{R}$ & $\mathrm{R}$ & $\mathrm{R}$ & $\mathrm{R}$ & $\mathrm{R}$ & 0.380 & YES & YES \\
\hline SL129 & & S & । & $\mathrm{R}$ & S & । & $\mathrm{R}$ & $\mathrm{R}$ & $\mathrm{R}$ & $\mathrm{R}$ & I & $\mathrm{R}$ & 0.190 & $\mathrm{~N} / \mathrm{D}$ & NO \\
\hline SL150 & & $\mathrm{R}$ & R & $\mathrm{R}$ & $S$ & 1 & I & $\mathrm{R}$ & $\mathrm{R}$ & $\mathrm{R}$ & $\mathrm{R}$ & R & 0.190 & YES & YES \\
\hline SL151 & & $\mathrm{R}$ & I & $\mathrm{R}$ & $S$ & $S$ & $\mathrm{R}$ & $\mathrm{R}$ & R & $\mathrm{R}$ & 1 & R & 0.380 & YES & YES \\
\hline SL153 & & R & R & $\mathrm{R}$ & 1 & 1 & $\mathrm{R}$ & $\mathrm{R}$ & $\mathrm{R}$ & $\mathrm{R}$ & 1 & $\mathrm{R}$ & 0.250 & YES & YES \\
\hline SL154 & & $\mathrm{R}$ & R & $\mathrm{R}$ & 1 & $\mathrm{~S}$ & $\mathrm{R}$ & $\mathrm{R}$ & $\mathrm{R}$ & $\mathrm{R}$ & 1 & $\mathrm{R}$ & 0.190 & N/D & NO \\
\hline SL155 & & R & R & $\mathrm{R}$ & S & $S$ & $\mathrm{R}$ & $\mathrm{R}$ & $\mathrm{R}$ & $\mathrm{R}$ & I & $\mathrm{R}$ & 0.250 & N/D & NO \\
\hline SL156 & & $\mathrm{R}$ & R & $\mathrm{R}$ & 1 & 1 & $\mathrm{R}$ & 1 & $\mathrm{R}$ & $\mathrm{R}$ & $\mathrm{R}$ & R & 0.250 & YES & YES \\
\hline SL157 & & R & R & $\mathrm{R}$ & $S$ & 1 & $\mathrm{R}$ & 1 & $\mathrm{R}$ & $\mathrm{R}$ & $\mathrm{R}$ & $\mathrm{R}$ & 0.250 & YES & YES \\
\hline SL170 & & $\mathrm{R}$ & 1 & $\mathrm{R}$ & $S$ & $S$ & $\mathrm{R}$ & $\mathrm{R}$ & R & $\mathrm{R}$ & 1 & R & 0.750 & YES & YES \\
\hline SL172 & & $\mathrm{R}$ & R & $\mathrm{R}$ & $S$ & $S$ & $S$ & $\mathrm{R}$ & $\mathrm{R}$ & $\mathrm{R}$ & $\mathrm{R}$ & I & 0.190 & YES & YES \\
\hline SL173 & & R & R & $\mathrm{R}$ & 1 & $S$ & $\mathrm{R}$ & $\mathrm{R}$ & $\mathrm{R}$ & $\mathrm{R}$ & 1 & R & 0.380 & YES & YES \\
\hline SL177 & & R & R & $\mathrm{R}$ & $S$ & S & $\mathrm{R}$ & $\mathrm{R}$ & $\mathrm{R}$ & $\mathrm{R}$ & I & $\mathrm{R}$ & 0.250 & YES & YES \\
\hline SL187 & & R & I & $\mathrm{R}$ & $S$ & $S$ & $S$ & 1 & R & $\mathrm{R}$ & I & I & 0.250 & YES & YES \\
\hline SL193 & & $\mathrm{R}$ & 1 & $\mathrm{R}$ & $S$ & $S$ & $\mathrm{R}$ & 1 & R & $\mathrm{R}$ & $S$ & $\mathrm{R}$ & 0.250 & YES & YES \\
\hline SL194 & & $\mathrm{R}$ & R & $\mathrm{R}$ & S & $S$ & $\mathrm{R}$ & $\mathrm{R}$ & $\mathrm{R}$ & $\mathrm{R}$ & 1 & $\mathrm{R}$ & 0.380 & N/D & NO \\
\hline SL197 & & $\mathrm{R}$ & $\mathrm{R}$ & $\mathrm{R}$ & S & $S$ & S & 1 & $\mathrm{R}$ & $\mathrm{R}$ & 1 & 1 & 0.250 & YES & YES \\
\hline SL200 & & $\mathrm{R}$ & R & $\mathrm{R}$ & S & 1 & $S$ & $\mathrm{R}$ & $\mathrm{R}$ & $\mathrm{R}$ & $\mathrm{R}$ & $\mathrm{R}$ & 0.190 & YES & YES \\
\hline SL202 & & $\mathrm{R}$ & $\mathrm{R}$ & $\mathrm{R}$ & S & I & S & $\mathrm{R}$ & $\mathrm{R}$ & $\mathrm{R}$ & $\mathrm{R}$ & $\mathrm{R}$ & 0.190 & YES & YES \\
\hline SL205 & & $\mathrm{R}$ & R & $\mathrm{R}$ & 1 & 1 & $S$ & $\mathrm{R}$ & $\mathrm{R}$ & $\mathrm{R}$ & $\mathrm{R}$ & 1 & 0.190 & YES & YES \\
\hline SL160 & Enterobacter cloacae & S & S & $S$ & S & S & $\mathrm{S}$ & S & $\mathrm{R}$ & $\mathrm{S}$ & $\mathrm{S}$ & S & 0.380 & NO & NO \\
\hline SL161 & & $\mathrm{R}$ & $\mathrm{R}$ & I & । & I & $\mathrm{R}$ & S & $\mathrm{R}$ & $\mathrm{R}$ & $\mathrm{R}$ & R & 0.250 & YES & YES \\
\hline SL167 & & I & । & I & S & S & $\mathrm{R}$ & 1 & $\mathrm{R}$ & $\mathrm{R}$ & 1 & $\mathrm{R}$ & 0.380 & YES & YES \\
\hline SL168 & & $\mathrm{R}$ & $\mathrm{R}$ & । & 1 & $S$ & $\mathrm{R}$ & I & $\mathrm{R}$ & $\mathrm{R}$ & I & $\mathrm{R}$ & 2.000 & YES & YES \\
\hline SL174 & & $\mathrm{R}$ & 1 & । & S & S & $\mathrm{R}$ & I & $\mathrm{R}$ & $\mathrm{R}$ & $\mathrm{R}$ & I & 0.380 & YES & YES \\
\hline SL175 & & I & I & S & S & S & $\mathrm{R}$ & S & $\mathrm{R}$ & $\mathrm{R}$ & $\mathrm{R}$ & $\mathrm{R}$ & 0.250 & YES & YES \\
\hline SL179 & & $\mathrm{R}$ & $\mathrm{R}$ & $\mathrm{R}$ & S & $S$ & $\mathrm{R}$ & 1 & R & $\mathrm{R}$ & $\mathrm{R}$ & $\mathrm{R}$ & 0.250 & YES & YES \\
\hline SL181 & & S & S & $S$ & S & S & $\mathrm{R}$ & $S$ & $\mathrm{R}$ & $\mathrm{R}$ & I & 1 & 0.380 & YES & YES \\
\hline SL182 & & R & R & I & I & $S$ & $\mathrm{R}$ & $S$ & $\mathrm{R}$ & $\mathrm{R}$ & 1 & $\mathrm{R}$ & 0.380 & YES & YES \\
\hline SL189 & & $S$ & S & I & $S$ & S & $\mathrm{R}$ & $S$ & R & $\mathrm{R}$ & 1 & 1 & 0.250 & YES & YES \\
\hline SL192 & & S & $S$ & I & $S$ & S & $\mathrm{R}$ & $\mathrm{R}$ & $\mathrm{R}$ & $\mathrm{R}$ & $S$ & $S$ & 0.380 & NO & NO \\
\hline SL195 & & I & 1 & $S$ & S & $S$ & $\mathrm{R}$ & $S$ & R & $\mathrm{R}$ & 1 & 1 & 0.380 & YES & YES \\
\hline SL198 & & $\mathrm{R}$ & $\mathrm{R}$ & $S$ & S & S & $\mathrm{R}$ & $S$ & R & $\mathrm{R}$ & 1 & $\mathrm{R}$ & 0.250 & YES & YES \\
\hline SL199 & & $\mathrm{R}$ & I & I & $S$ & $S$ & $\mathrm{R}$ & $S$ & $\mathrm{R}$ & $\mathrm{R}$ & 1 & 1 & 0.380 & YES & YES \\
\hline SL203 & & I & 1 & I & S & $S$ & $\mathrm{R}$ & $S$ & $\mathrm{R}$ & $\mathrm{R}$ & 1 & I & 0.380 & YES & YES \\
\hline SL115 & Escherichia coli & S & S & S & S & S & S & S & $S$ & $S$ & $\mathrm{~S}$ & S & 0.190 & NO & NO \\
\hline SL121 & & S & S & $\mathrm{R}$ & S & S & $\mathrm{R}$ & $\mathrm{R}$ & $\mathrm{R}$ & S & $S$ & I & 0.190 & NO & NO \\
\hline SL125 & & S & S & S & S & S & S & S & $S$ & S & $\mathrm{S}$ & S & 0.190 & NO & NO \\
\hline SL133 & & S & S & S & S & S & S & $\mathrm{R}$ & $\mathrm{R}$ & $S$ & $\mathrm{~S}$ & $\mathrm{R}$ & 0.190 & NO & NO \\
\hline SL137 & & S & S & S & S & S & S & $\mathrm{R}$ & $\mathrm{R}$ & S & $\mathrm{S}$ & $\mathrm{R}$ & 0.190 & NO & NO \\
\hline
\end{tabular}


Table 1 Antibiotic sensitivity testing results (Continued)

\begin{tabular}{|c|c|c|c|c|c|c|c|c|c|c|c|c|c|c|c|}
\hline SL152 & & $S$ & $S$ & 1 & $S$ & $S$ & R & $R$ & $\mathrm{R}$ & $R$ & $S$ & $S$ & 0.190 & NO & NO \\
\hline SL158 & & $S$ & $S$ & $R$ & 1 & $S$ & $R$ & $\mathrm{R}$ & $\mathrm{R}$ & $S$ & $S$ & R & 0.190 & NO & NO \\
\hline SL159 & & $S$ & $S$ & S & 1 & $S$ & S & S & $S$ & $S$ & S & S & 0.190 & $\mathrm{NO}$ & $\mathrm{NO}$ \\
\hline SL169 & & $S$ & $S$ & $S$ & $S$ & $S$ & $\mathrm{R}$ & R & $\mathrm{R}$ & $S$ & $S$ & $S$ & 0.250 & $\mathrm{NO}$ & NO \\
\hline SL171 & & $S$ & $S$ & $S$ & $S$ & $S$ & $\mathrm{R}$ & 1 & $\mathrm{R}$ & $S$ & $S$ & R & 0.250 & $\mathrm{NO}$ & $\mathrm{NO}$ \\
\hline SL176 & & $S$ & $\mathrm{~S}$ & $\mathrm{R}$ & $S$ & $S$ & $S$ & $S$ & $\mathrm{R}$ & $S$ & $S$ & I & 0.250 & $\mathrm{NO}$ & $\mathrm{NO}$ \\
\hline SL178 & & $\mathrm{R}$ & $S$ & $S$ & $S$ & $S$ & $\mathrm{R}$ & I & R & 1 & $S$ & $S$ & 0.250 & $\mathrm{NO}$ & NO \\
\hline SL188 & & S & $S$ & $S$ & $S$ & $S$ & $\mathrm{R}$ & 1 & R & $S$ & $S$ & $S$ & 0.250 & $\mathrm{NO}$ & $\mathrm{NO}$ \\
\hline SL166 & Escherichia hermannii & $\mathrm{R}$ & $\mathrm{R}$ & 1 & S & S & $\mathrm{R}$ & S & $\mathrm{R}$ & $\mathrm{R}$ & I & $\mathrm{R}$ & 0.190 & YES & YES \\
\hline SL108 & Klebsiella pneumoniae & S & $S$ & 1 & $S$ & $S$ & S & S & S & $S$ & $S$ & $S$ & 0.250 & NO & NO \\
\hline SL113 & & $S$ & S & I & S & $S$ & $\mathrm{R}$ & । & $\mathrm{R}$ & $\mathrm{R}$ & $\mathrm{R}$ & $S$ & 0.250 & YES & YES \\
\hline SL116 & & S & S & 1 & $S$ & S & S & S & S & S & S & S & 0.190 & NO & NO \\
\hline SL136 & & s & S & S & $S$ & $\mathrm{~S}$ & R & S & $\mathrm{R}$ & $\mathrm{S}$ & S & $S$ & 0.250 & NO & NO \\
\hline SL141 & & s & S & $S$ & S & $\mathrm{S}$ & S & S & S & S & S & S & 0.250 & NO & NO \\
\hline SL162 & & $\mathrm{R}$ & $\mathrm{R}$ & $\mathrm{R}$ & I & I & $\mathrm{R}$ & R & $\mathrm{R}$ & $R$ & 1 & R & 0.190 & YES & YES \\
\hline SL163 & & $\mathrm{R}$ & $\mathrm{R}$ & 1 & 1 & 1 & $\mathrm{R}$ & $\mathrm{R}$ & R & R & $S$ & R & 0.190 & YES & YES \\
\hline SL164 & & $S$ & $S$ & 1 & $S$ & $S$ & $\mathrm{R}$ & $\mathrm{R}$ & $\mathrm{R}$ & R & $S$ & R & 0.190 & YES & YES \\
\hline SL165 & & 1 & 1 & 1 & $S$ & $S$ & $\mathrm{R}$ & $\mathrm{R}$ & $\mathrm{R}$ & $\mathrm{R}$ & I & R & 0.250 & YES & YES \\
\hline SL180 & & $S$ & $S$ & $S$ & $S$ & $S$ & $\mathrm{R}$ & S & $\mathrm{R}$ & $\mathrm{R}$ & I & I & 0.380 & YES & YES \\
\hline SL183 & & $S$ & $S$ & $S$ & $S$ & $S$ & $\mathrm{R}$ & $R$ & $\mathrm{R}$ & $S$ & $S$ & I & 0.250 & $\mathrm{NO}$ & NO \\
\hline SL185 & & 1 & I & R & $S$ & $S$ & $S$ & I & R & $\mathrm{R}$ & $\mathrm{R}$ & 1 & 0.190 & YES & YES \\
\hline SL186 & & 1 & 1 & 1 & $S$ & $\mathrm{~S}$ & $\mathrm{R}$ & R & R & R & $S$ & R & 0.250 & YES & YES \\
\hline SL190 & & $S$ & $S$ & $R$ & 1 & 1 & $\mathrm{R}$ & $R$ & $\mathrm{R}$ & $S$ & $\mathrm{R}$ & $\mathrm{R}$ & 0.250 & NO & NO \\
\hline SL196 & & $\mathrm{R}$ & $\mathrm{R}$ & $\mathrm{R}$ & 1 & 1 & $S$ & 1 & $R$ & $\mathrm{R}$ & $\mathrm{R}$ & 1 & 0.250 & YES & YES \\
\hline SL184 & Enterobacter sp/Leclercia sp. & । & । & $\mathrm{R}$ & S & S & $\mathrm{R}$ & S & $\mathrm{R}$ & $\mathrm{R}$ & । & I & 0.250 & YES & YES \\
\hline SL191 & & 1 & $\mathrm{R}$ & $\mathrm{R}$ & 1 & 1 & $\mathrm{R}$ & S & $\mathrm{R}$ & $\mathrm{R}$ & I & I & 0.250 & YES & YES \\
\hline SL201 & & $R$ & $\mathrm{R}$ & $\mathrm{R}$ & $\mathrm{R}$ & । & $\mathrm{R}$ & S & $\mathrm{R}$ & $\mathrm{R}$ & $\mathrm{R}$ & 1 & 0.250 & YES & YES \\
\hline SL204 & & R & $\mathrm{R}$ & 1 & R & I & R & R & $\mathrm{R}$ & $\mathrm{R}$ & $\mathrm{R}$ & I & 0.190 & YES & YES \\
\hline \multicolumn{16}{|c|}{ Environmental isolates } \\
\hline SL512 & Enterobacter cloacae & S & S & S & 1 & $\mathrm{~S}$ & S & R & $\mathrm{R}$ & S & S & $\mathrm{S}$ & 0.250 & NO & NO \\
\hline SL532 & & I & I & S & S & S & $\mathrm{R}$ & S & $\mathrm{R}$ & $\mathrm{R}$ & $\mathrm{R}$ & $\mathrm{R}$ & N/A & YES & YES \\
\hline SL513 & Klebsiella pneumoniae & $\mathrm{R}$ & $\mathrm{R}$ & S & S & $\mathrm{S}$ & S & S & $\mathrm{R}$ & $\mathrm{R}$ & । & $\mathrm{R}$ & 0.190 & YES & YES \\
\hline SL531 & & S & $S$ & $S$ & $S$ & S & S & S & $S$ & $S$ & $S$ & S & 0.190 & NO & NO \\
\hline SL522 & Pantoea dispersa & S & $S$ & S & S & $S$ & S & S & $S$ & $S$ & S & $S$ & 0.250 & NO & $\mathrm{NO}$ \\
\hline
\end{tabular}

${ }^{a}$ Consensus identification based on combination phenotypic (API20E/ID32E) and 16S rRNA and rpoB gene sequencing

${ }^{b}$ Disk diffusion testing using the following antibiotics: ATM aztreonam, CAZ ceftazidime, CIP ciprofloxacin, IMP imipenem, TGC tigecycline, C chloramphenicol, $D$ doxycycline, $G$ sulfisoxazole, $G M$ gentamicin, $K$ kanamycin, $S A M$ ampicillin/sulbactam. $R$ resistant, $I$ intermediate, and $S$ sensitive phenotypes were determined based on CLSI criteria

${ }^{\mathrm{C}}$ ESBL phenotypes: YES - ESBL detected, NO - no ESBL present, N/D - ESBL presence could not be determined based on the E-test or double disk diffusion assay

\section{Urine isolate antibiotic susceptibility}

The 70 urine isolates were tested for their susceptibility to 11 antibiotics or antibiotic combinations covering six classes of antimicrobial compounds and an ESBL phenotype (Table 1). Overall, $85.7 \%$ of the clinical isolates were identified as MDR. More than $90 \%$ of the urinary isolates were resistant to sulphonamides. Large proportions of the isolates were also found to be resistant to gentamycin (72.9\%), chloramphenicol (72.9\%), ampicillin/sulbactam (51.4\%), and/or ciprofloxacin (47.1\%). A considerable proportion of the clinical isolates (38.6\%) also demonstrated high resistance to the third generation cephalosporin, ceftazidime, which is typically indicative of ESBL production but may be due to inducible AmpC enzymes common in C. freundii and Enterobacter spp. Indeed, testing for the presence of ESBLs revealed 
that $64.3 \%$ of the urine isolates produced an ESBL as defined by CLSI criteria. PCR-based detection of $b l a_{\text {CTX-M }}$ genes in every isolate presenting an ESBL phenotype (results not shown) confirmed the widespread presence of these genes and potentially enzymes in the analyzed isolate collection. In spite of the overall non-susceptibility to the majority of antimicrobials tested, none of the strains were resistant to tigecycline, and only two strains were resistant to imipenem. However, the two strains resistant to imipenem by disk diffusion - as well as all classified as having intermediate sensitivity - were deemed susceptible by CLSI standards when MICs were determined by E-test.

There appeared to be substantial species-specific differences in antimicrobial resistance with $C$. freundii being the most highly MDR species followed by Enterobacter sp./Leclercia sp., E. hermannii, E. cloacae, K. pneumoniae and E. coli (Table 2). All of the C. freundii urine isolates were resistant to sulfisoxazole, gentamycin and ciprofloxacin and most were resistant to several other antibiotics: the average $C$. freundii isolate was resistant to $>7$ antibiotics out of the 11 tested. In addition, $81.8 \%$ of the C. freundii isolates produced ESBLs. The presence or absence of ESBLs in four isolates (SL129,
SL154, SL155 and SL194) could not be determined based on phenotype only. Double disk synergy tests indicated the possible presence of an inhibitor-resistant $\beta$ lactamase in these strains (data not shown).

Fourteen of the $15 \mathrm{E}$. cloacae isolates $(93.3 \%)$ were MDR, with 13 producing an ESBL and showing resistance for sulfisoxazole and a diminished sensitivity to at least five other antibiotics (with chloramphenicol, gentamycin, and kanamycin being among the most prevalent). The majority of $K$. pneumoniae isolates $(73.3 \%)$ were also MDR; most were insensitive to at least five antibiotics including chloramphenicol, doxycycline, gentamicin and a combination of ampicillin with sulbactam. Over half exhibited an ESBL phenotype, but a significant proportion (26.7 \%) were sensitive to ciprofloxacin. All four Enterobacter sp./Leclercia sp. strains that were isolated from the urine cultures were positive for ESBL production and non-susceptible to eight or more antibiotics. One strain tested resistant or intermediate to all 11 antibiotics tested by disk diffusion. However, imipenem resistance and intermediate resistance in these isolates were not confirmed by E-test. The single E. hermannii identified among the urinary isolates presented a phenotype similar to the

Table 2 Percent prevalence of antimicrobial sensitivity phenotypes in urine isolates as determined by the disk diffusion method

\begin{tabular}{|c|c|c|c|c|c|c|c|c|c|c|c|c|c|c|}
\hline \multirow[t]{2}{*}{ Species } & \multirow[t]{2}{*}{ No. } & \multirow[t]{2}{*}{ Phenotype } & \multicolumn{11}{|c|}{ Antibiotic $^{a}$} & \multirow[t]{2}{*}{ ESBL } \\
\hline & & & ATM & CAZ & CIP & IMP & TGC & C & D & G & GM & K & SAM & \\
\hline \multirow[t]{3}{*}{ Citrobacter freundii } & \multirow[t]{3}{*}{22} & $\mathrm{R}$ & 95.5 & 68.2 & 100.0 & 0.0 & 0.0 & 68.2 & 77.3 & 100.0 & 100.0 & 45.5 & 81.8 & \multirow[t]{3}{*}{81.8} \\
\hline & & I & 0.0 & 31.8 & 0.0 & 22.7 & 40.9 & 4.5 & 22.7 & 0.0 & 0.0 & 50.0 & 18.2 & \\
\hline & & S & 4.5 & 0.0 & 0.0 & 77.3 & 59.1 & 27.3 & 0.0 & 0.0 & 0.0 & 4.5 & 0.0 & \\
\hline \multirow[t]{3}{*}{ Enterobacter sp./Leclercia sp. } & \multirow[t]{3}{*}{4} & R & 50.0 & 75.0 & 75.0 & 50.0 & 0.0 & 100.0 & 25.0 & 100.0 & 100.0 & 50.0 & 0.0 & \multirow[t]{3}{*}{100.0} \\
\hline & & I & 50.0 & 25.0 & 25.0 & 25.0 & 75.0 & 0.0 & 0.0 & 0.0 & 0.0 & 50.0 & 100.0 & \\
\hline & & S & 0.0 & 0.0 & 0.0 & 25.0 & 25.0 & 0.0 & 75.0 & 0.0 & 0.0 & 0.0 & 0.0 & \\
\hline \multirow[t]{3}{*}{ Escherichia hermannii } & \multirow[t]{3}{*}{1} & R & 100.0 & 100.0 & 0.0 & 0.0 & 0.0 & 100.0 & 0.0 & 100.0 & 100.0 & 0.0 & 100.0 & \multirow[t]{3}{*}{100.0} \\
\hline & & I & 0.0 & 0.0 & 100.0 & 0.0 & 0.0 & 0.0 & 0.0 & 0.0 & 0.0 & 100.0 & 0.0 & \\
\hline & & S & 0.0 & 0.0 & 0.0 & 100.0 & 100.0 & 0.0 & 100.0 & 0.0 & 0.0 & 0.0 & 0.0 & \\
\hline \multirow[t]{3}{*}{ Enterobacter cloacae } & \multirow[t]{3}{*}{15} & $\mathrm{R}$ & 46.7 & 33.3 & 6.7 & 0.0 & 0.0 & 93.3 & 6.7 & 100.0 & 93.3 & 26.7 & 46.7 & \multirow[t]{3}{*}{86.7} \\
\hline & & । & 26.7 & 40.0 & 60.0 & 20.0 & 6.7 & 0.0 & 26.7 & 0.0 & 0.0 & 60.0 & 40.0 & \\
\hline & & S & 26.7 & 26.7 & 33.3 & 80.0 & 93.3 & 6.7 & 66.7 & 0.0 & 6.7 & 13.3 & 13.3 & \\
\hline \multirow[t]{3}{*}{ Klebsiella pneumoniae } & \multirow[t]{3}{*}{15} & $\mathrm{R}$ & 20.0 & 20.0 & 26.7 & 0.0 & 0.0 & 66.7 & 46.7 & 80.0 & 60.0 & 26.7 & 40.0 & \multirow[t]{3}{*}{60.0} \\
\hline & & I & 20.0 & 20.0 & 46.7 & 26.7 & 26.7 & 0.0 & 20.0 & 0.0 & 0.0 & 20.0 & 26.7 & \\
\hline & & S & 60.0 & 60.0 & 26.7 & 73.3 & 73.3 & 33.3 & 33.3 & 20.0 & 40.0 & 53.3 & 33.3 & \\
\hline \multirow[t]{3}{*}{ Escherichia coli } & \multirow[t]{3}{*}{13} & $\mathrm{R}$ & 7.7 & 0.0 & 23.1 & 0.0 & 0.0 & 53.8 & 46.2 & 76.9 & 7.7 & 0.0 & 30.8 & \multirow[t]{3}{*}{0.0} \\
\hline & & I & 0.0 & 0.0 & 7.7 & 15.4 & 0.0 & 0.0 & 23.1 & 0.0 & 7.7 & 0.0 & 15.4 & \\
\hline & & S & 92.3 & 100.0 & 69.2 & 84.6 & 100.0 & 46.2 & 30.8 & 23.1 & 84.6 & 100.0 & 53.8 & \\
\hline \multirow[t]{3}{*}{ Overall } & \multirow[t]{3}{*}{70} & $R$ & 50.0 & 38.6 & 47.1 & 2.9 & 0.0 & 72.9 & 45.7 & 91.4 & 72.9 & 28.6 & 51.4 & \multirow[t]{3}{*}{62.9} \\
\hline & & I & 12.9 & 24.3 & 27.1 & 21.4 & 24.3 & 1.4 & 21.4 & 0.0 & 1.4 & 37.1 & 28.6 & \\
\hline & & S & 37.1 & 37.1 & 25.7 & 75.7 & 75.7 & 25.7 & 32.9 & 8.6 & 25.7 & 34.3 & 20.0 & \\
\hline
\end{tabular}

Disk diffusion testing using the following antibiotics: ATM aztreonam, CAZ ceftazidime, CIP ciprofloxacin, IMP imipenem, TGC tigecycline, C chloramphenicol, $D$ doxycycline, $G$ sulfisoxazole, $G M$ gentamicin, $K$ kanamycin, $S A M$ ampicillin/sulbactam. $R$ resistant, I intermediate, and $S$ sensitive phenotypes were determined based on CLSI criteria 
Enterobacter sp./Leclercia sp.: it exhibited ESBL production and resistance or intermediate resistance to all of the tested antibiotics with the exception of doxycycline, imipenem and tigecycline. Surprisingly, the E. coli strains were found to be the least resistant of all of the urine isolates tested and did not demonstrate the ESBL phenotype. Nevertheless, $61.5 \%$ of the E. coli isolates could be classified as MDR and were most commonly resistant to sulfisoxazole, chloramphenicol and doxycycline.

\section{Hospital environment isolate antibiotic susceptibility}

The same panel of 11 antibiotics were used for susceptibility testing of the five Enterobacteriaceae and 26 nonEnterobacteriaceae isolates from hospital fomites (Table 1). Two of the five Enterobacteriaceae isolates (K. pneumoniae SL513 and E. cloacae SL532) were MDR and carried ESBLs (Table 1). Of the three other Enterobacteriaceae isolates, E. cloacae SL512 was resistant to only sulfisoxazole and doxycycline and was sensitive to all of the other antibiotics tested. The remaining two isolates, $K$. pneumoniae SL531 and Pantoea dispersa SL552, were sensitive to all of the antibiotics tested.

Breakpoint thresholds have been published for Staphylococcus spp. for only seven of the 11 antibiotics used for susceptibility testing and for none of the 11 antibiotics used for testing Bacillus spp. For this reason, only the zone diameters of the 26 non-Enterobacteriaceae isolates identified as Staphylococcus spp. and Bacillus spp. are shown in Additional file 3: Table S3. Of the seven antibiotics for which Staphylococcus sp. breakpoints have been published, the tested Staphylococcus isolates were all deemed susceptible.

\section{Discussion}

E. coli typically account for $\sim 80 \%$ of the bacteria isolated from uncomplicated UTI urines, with non-E. coli Enterobacteriaceae (e.g., Klebsiella spp., Enterococcus spp., Citrobacter spp.) usually present at much lower levels $[27,28]$. This study was specifically designed to analyze the resistance patterns of non-E. coli Enterobacteriaceae. While we did not determine the overall prevalence of $E$. coli in the cultured urine samples, we collected all nonE. coli Enterobacteriaceae and were surprised to find that they were present in such a large proportion of the tested samples $(57.0 \%)$. Indeed, both $K$. pneumoniae and E. cloacae were cultured from $16.1 \%$ of the urine samples - levels more typical of complicated UTIs or diabetic patients than general outpatient populations $[28,29]$. It was particularly unexpected that $C$. freundii was isolated from almost a quarter (23.6 \%) of the collected urine samples, accounting for $38.6 \%$ of all non-E.coli isolates. While surprising, our findings are in agreement with a number of recent studies that have reported on the increased incidence of Citrobacter among urinary pathogens in developing countries [30-32], thus suggesting that Citrobacter may be becoming an increasingly important emerging urinary tract pathogen in resource-limited settings [33]. The high percentage of the non-E. coli UTIassociated bacteria analyzed in this study may also indicate weakened immune systems or additional health problems in the studied population. However, it is important to bear in mind that due to the relatively short duration of the study and small size of the sample, the observed proportions of pathogens recovered from urine samples may not be representative for the wider population of Bo and not be typical over longer periods of time.

Overall, data on antibiotic resistance in the Enterobacteriaceae from countries in West Africa (with the great majority originating from Nigeria and Senegal) are scarce and show enormous variation depending on the particular population and study design [12, 34]. For example, the prevalence of the ESBL phenotype in Enterobacteriaceae have been reported to vary from $10 \%$ in diarrheal samples in Senegal to $96 \%$ in Mali [34]. With respect to Sierra Leone however, there is an almost complete lack of information on bacterial antibiotic resistance.

The analysis of urine isolates used in this study suggests that clinically relevant MDR bacteria are prevalent in the studied outpatient population. Especially concerning is the high percentage of ESBL carriage among the analyzed non-E. coli Enterobacteriaceae, confirmed by both phenotypic and molecular assays. The chemotherapeutic options for the treatment of infections caused by these currently circulating Enterobacteriaceae strains are limited. Especially worrisome is the fact that a large proportion of these strains were not only resistant to first-line antibiotics such as sulphonamides (trimethoprim-sulfonamide is among the most commonly used treatments for uncomplicated UTIs [35]), ß-lactams and chloramphenicol, but many of them were also resistant to such second-line drugs as aminoglycosides and broad spectrum antibiotics such as the fluoroquinolone ciprofloxacin. While the high percentage of MDR isolates observed in this collection may be partly explained by the study design and overrepresentation of species rich in intrinsic resistance mechanisms (e.g. Citrobacter sp., Enterobacter sp.), even the usually less multiresistant species such as E. coli were found to contain significant proportion of MDR strains. On the other hand, despite earlier reports describing the detection of carbapenemase (i.e., $b l a_{\mathrm{VIM}-1}, b l a_{\mathrm{DIM}-1}, b l a_{\mathrm{OXA}-51}$ and $b l a_{\mathrm{OXA}-58}$ ) and tigecycline $(\operatorname{tet}(X))$ resistance genes in this community $[14,36]$, there was no evidence for the spread of phenotypic resistance to these last-line antibiotics.

The contamination of the hospital surfaces is usually considered in the context of increased risk for hospital 
acquired UTIs and spread between inpatients or healthcare workers and patients. Mercy Hospital serves mostly the needs of an outpatient population (30-50 patients per day) with a limited number of inpatients (24 beds with varying occupancy). Many outpatients who spend considerable amounts of time in waiting rooms and other areas of the hospital, are exposed to hospital fomites and have contact with staff serving both outpatient and inpatient populations. This study made an attempt to determine the role of hospital environment fomites in the spread of MDR Enterobacteriaceae in Mercy Hospital. Previous studies have shown that bacterial pathogens, including members of Enterobacteriaceae such as E. coli and K. pneumoniae, are capable of surviving from days to over a year on dry, inanimate surfaces [37, 38]. Screening the environmental samples collected at Mercy Hospital demonstrated that the majority of isolates producing dark blue colonies on the chromogenic medium were Bacillus sp. Only five of the isolates collected from hospital surfaces $(2.7 \%)$ were identified as members of the Enterobacteriaceae. These results suggest that fomites in the Mercy Hospital open air ventilated environment are not a major source of pathogenic Enterobacteriaceae and that the observed resistance among the outpatient urinary isolates was likely not acquired from hospital fomite reservoirs. However, while relatively rare in the hospital environment, some of these environmental isolates demonstrated MDR phenotype and carried ESBLs. These observations warrant vigilance and the implementation of hospital hygiene procedures to maintain low levels of fomite contamination and potentially prevent the wider spread of these MDR pathogens.

\section{Conclusions}

The surprisingly high incidence of Citrobacter found among urine isolates in the analyzed Mercy Hospital outpatient population suggests that Citrobacter may be becoming an increasingly important emerging urinary tract pathogen in this particular resource-limited setting. The high percentage of other non-E. coli Enterobacteriaceae among pathogens found in the urine of these patients may also indicate the prevalence of additional health problems in the studied population. Antibiotic susceptibility testing results show that clinically relevant MDR Enterobacteriaceae are prevalent in the studied population, which may reflect the lack of the national antibiotic stewardship policies in Sierra Leone and widespread access to antimicrobials without prescription. The results of hospital environment sampling showing very low level of contamination with MDR Enterobacteriaceae suggest that fomites are not a likely source of these pathogens in Mercy Hospital.

\section{Ethics approval and consent to participate}

While there are no Human Subject Regulations in Sierra Leone, all the samples used in this study were collected in a bigger study approved by both Sierra Leone Ethics and Scientific Review Committee and the Njala University Institutional Review Board. All participants of that study or their parents/guardians provided informed written or verbal consent to be included in the study. As pre-existing diagnostic samples, these isolates were stripped of all personal identifiers and exempt from consideration as Human Subject Research as defined by 32 CFR 219, Section 101b in the US.

\section{Consent for publication \\ Not applicable.}

\section{Availability of data and materials}

All the data discussed in this manuscript are included in the manuscript or the accompanying additional/supplementary files. All the described bacterial isolates are available from the authors of the manuscript upon request and in compliance with local and international regulations related to transferring infectious biological samples.

\section{Additional files}

Additional file 1: Table S1. Hospital environment sampling information. Information on the sources and dates of collection of hospital environmental samples. (XLS $30 \mathrm{~kb}$ )

Additional file 2: Table S2. Full results of isolate identification. The full results of identification of isolates included in this study, including full results of phenotypic and molecular identification assays. (XLSX 51 kb)

Additional file 3: Table S3. Sensitivity testing results of nonEnterobacteriaceae environmental isolates. Results of sensitivity testing of environmental isolates other than Enterobacteriaceae using disk diffusion method. (XLSX $62 \mathrm{~kb}$ )

\section{Abbreviations}

ATM: aztreonam; C: chloramphenicol; CAZ: ceftazidime; CIP: ciprofloxacin; CLSI: Clinical and Laboratory Standards Institute; CRE: carbapenem resistant Enterobacteriaceae; CT/CTL: cefotaxime/cefotaxime + clavulanic acid; D: doxycycline; ESBL: extended spectrum $\beta$-lactamase; G: sulfisoxazole; GM: gentamicin; IMP: imipenem; K: kanamycin; MDR: multi-drug resistant; MHA: Mueller Hinton agar; MIC: minimum inhibitory concentration; PCR: polymerase chain reaction; SAM: ampicillin with sulbactam; TGC: tigecycline; TZ/TZL: ceftazidime/ceftazidime + clavulanic acid; UTI: urinary tract infection.

\section{Competing interests}

The authors declare that they have no competing interests.

\footnotetext{
Authors' contributions

TAL and CRT were involved in the study conception, clinical isolate collection, isolate identification, susceptibility testing, and drafting of the manuscript. UB and RA were involved in clinical isolate collection, environmental isolate collection, isolate identification, susceptibility testing and manuscript revision. MGS was involved in performing isolate identification using molecular methods and manuscript revision. WHC performed the testing to confirm the presence of the CTX-M genes and was involved in manuscript revision. GJV was involved in the study conception, data analysis, and manuscript revision. DAS was involved in study conception and manuscript revision. All authors have read and approved of the final manuscript.
} 


\section{Acknowledgments}

Not applicable.

\section{Funding}

This work was supported in part by the Joint Science and Technology Office (JSTO), Defense Threat Reduction Agency (DTRA) and by the Office of Naval Research (ONR) via U.S. Naval Research Laboratory core funds. The funding entities had no role in or any influence over the design of the study, collection, analysis, and interpretation of data and the final manuscript.

\section{Author details}

'Naval Research Laboratory, Code 69104555 Overlook Avenue SW, Washington, DC 20375, USA. ${ }^{2}$ Mercy Hospital Research Laboratory, Bo, Sierra Leone. ${ }^{3}$ Liverpool School of Tropical Medicine, Liverpool, UK. ${ }^{4}$ Njala University, Bo, Sierra Leone. ${ }^{5}$ Bowie State University, Bowie, Maryland, USA.

\section{Received: 30 May 2015 Accepted: 6 April 2016}

\section{Published online: 18 April 2016}

\section{References}

1. Anonymous. The antibiotic alarm. Nature. 2013;495(7440):141.

2. Centers for Disease Control and Prevention. Antibiotic Resistance Threats in the United States, 2013. 2013. http://www.cdc.gov/drugresistance/threatreport-2013/. Accessed 28 May 2015

3. Snitkin ES, Zelazny AM, Thomas PJ, Stock F, NCSP group, Henderson DK, et al. Tracking a hospital outbreak of carbapenem-resistant Klebsiella pneumoniae with whole-genome sequencing. Sci Transl Med. 2012;4(148): 148ra16. doi:10.1126/scitransImed.3004129.

4. Escobar Perez JA, Olarte Escobar NM, Castro-Cardozo B, Valderrama Marquez IA, Garzon Aguilar MI, Martinez de la Barrera L, et al. Outbreak of NDM-1-producing Klebsiella pneumoniae in a neonatal unit in Colombia. Antimicrob Agents Chemother. 2013;57(4):1957-60. doi:10.1128/AAC.01447-12.

5. Bradford PA. Extended-spectrum beta-lactamases in the 21st century: characterization, epidemiology, and detection of this important resistance threat. Clin Microbiol Rev. 2001;14(4):933-51. doi:10.1128/CMR.14.4.933-951.2001.

6. Ben-Ami R, Rodriguez-Bano J, Arslan H, Pitout JD, Quentin C, Calbo ES, et al. A multinational survey of risk factors for infection with extended-spectrum beta-lactamase-producing Enterobacteriaceae in nonhospitalized patients. Clin Infect Dis. 2009:49(5):682-90. doi:10.1086/604713.

7. Perez F, Van Duin D. Carbapenem-resistant Enterobacteriaceae: a menace to our most vulnerable patients. Cleve Clin J Med. 2013;80(4):225-33. doi:10.3949/ccjm.80a.12182

8. Brolund A. Overview of ESBL-producing Enterobacteriaceae from a Nordic perspective. Infect Ecol Epidemiol. 2014;4. doi:10.3402/iee.v4.24555

9. Nordmann P, Naas T, Poirel L. Global spread of carbapenemase-producing Enterobacteriaceae. Emerg Infect Dis. 2011;17(10):1791-8. doi:10.3201/ eid1710.110655

10. Tada T, Miyoshi-Akiyama T, Dahal RK, Mishra SK, Ohara H, Shimada K, et al. Dissemination of multidrug-resistant Klebsiella pneumoniae clinical isolates with various combinations of carbapenemases (NDM-1 and OXA-72) and 16S rRNA methylases (ArmA, RmtC and RmtF) in Nepal. Int J Antimicrob Agents. 2013;42(4):372-4. doi:10.1016/j.jjantimicag.2013.06.014

11. Leski T, Vora G, Taitt CR. Multidrug resistance determinants from NDM-1 producing Klebsiella pneumoniae, United States of America. Int J Antimicrob Agents. 2012;40(3):282-4

12. World Health Organization. Antimicrobial resistance: global report on surveillance 2014. 2014. http://www.who.int/drugresistance/documents/ surveillancereport/en/. Accessed 27 May 2015

13. Tansarli GS, Athanasiou S, Falagas ME. Evaluation of antimicrobial susceptibility of Enterobacteriaceae causing urinary tract infections in Africa. Antimicrob Agents Chemother. 2013;57(8):3628-39. doi:10.1128/AAC.00359-13.

14. Leski TA, Bangura U, Jimmy DH, Ansumana R, Lizewski SE, Stenger DA, et al. Multidrug-resistant tet $(X)$-containing hospital isolates in Sierra Leone. Int J Antimicrob Agents. 2013;42(1):83-6. doi:10.1016/j.jjantimicag.2013.04.014.

15. Laxminarayan R, Duse A, Wattal C, Zaidi AKM, Wertheim HFL, Sumpradit N, et al. Antibiotic resistance - the need for global solutions. Lancet Infect Dis. 2013;13(12):1057-98. doi:10.1016/S1473-3099(13)70318-9.

16. Sosa AJ, Byarugaba DK, Amabile-Cuevas CF, Shueh P-R, Kariuki S, Okeke IN. Antimicrobial Resistance in Developing Countries. New York: Springer; 2010.
17. Kelesidis T, Kelesidis I, Rafailidis PI, Falagas ME. Counterfeit or substandard antimicrobial drugs: a review of the scientific evidence. J Antimicrob Chemother. 2007;60(2):214-36. doi:10.1093/jac/dkm109.

18. Okeke IN, Aboderin OA, Byarugaba DK, Ojo KK, Opintan JA. Growing problem of multidrug-resistant enteric pathogens in Africa. Emerg Infect Dis. 2007;13(11):1640-6. doi:10.3201/eid1311.070674.

19. Ansumana R, Malanoski AP, Bockarie AS, Sundufu AJ, Jimmy DH, Bangura $U$, et al. Enabling methods for community health mapping in developing countries. Int J Health Geogr. 2010;9:56. doi:10.1186/1476-072X-9-56.

20. Kommedal O, Kvello K, Skjastad R, Langeland N, Wiker HG. Direct 165 rRNA gene sequencing from clinical specimens, with special focus on polybacterial samples and interpretation of mixed DNA chromatograms. J Clin Microbiol. 2009;47(11):3562-8. doi:10.1128/JCM.00973-09.

21. Wang Q, Garrity GM, Tiedje JM, Cole JR. Naïve Bayesian classifier for rapid assignment of rRNA sequences into the new bacterial taxonomy. Appl Environ Microbiol. 2007;73(16):5261-7. doi:10.1128/AEM.00062-07.

22. Mollet $C$, Drancourt M, Raoult D. rpoB sequence analysis as a novel basis for bacterial identification. Mol Microbiol. 1997;26(5):1005-11.

23. Tayeb LA, Lefevre M, Passet V, Diancourt L, Brisse S, Grimont PA. Comparative phylogenies of Burkholderia, Ralstonia, Comamonas, Brevundimonas and related organisms derived from rpoB, gyrB and rrs gene sequences. Res Microbiol. 2008;159(3):169-77. doi:10.1016/j.resmic.2007.12.005.

24. Clinical and Laboratory Standards Institute. Performance Standards for Antimicrobial Susceptibility Testing: Nineteenth Informational Supplement M100-S19. 2009.

25. Food and Drug Administration. Tygacil, label information. 2013. http://www. accessdata.fda.gov/drugsatfda_docs/label/2013/021821 s026s031lbl.pdf. Accessed 27 May 2015

26. Fang $H$, Ataker F, Hedin G, Dornbusch K. Molecular epidemiology of extended-spectrum beta-lactamases among Escherichia coli isolates collected in a Swedish hospital and its associated health care facilities from 2001 to 2006. J Clin Microbiol. 2008;46(2):707-12. doi:10.1128/JCM.01943-07.

27. Ronald $A$. The etiology of urinary tract infection: traditional and emerging pathogens. Dis Mon. 2003;49(2):71-82. doi:10.1067/mda.2003.8.

28. Sheerin NS. Urinary tract infection. Medicine. 2011;39(7):384-9.

29. Salvatore S, Salvatore S, Cattoni E, Siesto G, Serati M, Psorice P, et al. Urinary tract infections in women. Eur J Obstet Gynecol Reprod Biol. 2011;156:131-6.

30. Metri BC, Jyothi P, Peerapur BV. Antibiotic resistance in Citrobacter spp. isolated from urinary tract infection. Urol Ann. 2013;5(4):312-3. doi:10.4103/0974-7796.120295.

31. Baral P, Neupane S, Marasini BP, Ghimire KR, Lekhak B, Shrestha B. High prevalence of multidrug resistance in bacterial uropathogens from Kathmandu, Nepal. BMC Res Notes. 2012;5:38. doi:10.1186/1756-0500-5-38.

32. Hanley J, Branford I, Gugnani HC, Wilkinson C, Uhrin T. Urinary bacterial pathogens and their antimicrobial susceptibility profile for the years 2005-2007 in St Kitts. West Indian Med J. 2009;58(6):571-4.

33. Ranjan KP, Ranjan N. Citrobacter: An emerging health care associated urinary pathogen. Urol Ann. 2013;5(4):313-4.

34. Storberg V. ESBL-producing Enterobacteriaceae in Africa - a non-systematic literature review of research published 2008-2012. Infect Ecol Epidemiol. 2014; 4. doi:10.3402/iee.v4.20342

35. Gillbert DN, Chambers HF, Eliopoulos GM, Saag MS. The Sanford Guide to Antimicrobial Therapy 2014. 44th ed. Sperryville: Antimicrobial Therapy, Inc.; 2014.

36. Leski TA, Bangura U, Jimmy DH, Ansumana R, Lizewski SE, Li RW, et al. Identification of bla $a_{\mathrm{OXA}-51-\text { like, }}$ bla OXA-58, $b a_{\mathrm{DIM}-1,}$, and blavIM carbapenemase genes in hospital Enterobacteriaceae isolates from Sierra Leone. J Clin Microbiol. 2013;51(7):2435-8. doi:10.1128/JCM.00832-13.

37. Hota B. Contamination, disinfection, and cross-colonization: are hospital surfaces reservoirs for nosocomial infection? Clin Infect Dis. 2004;39(8):1182-9. doi:10.1086/424667.

38. Kramer A, Schwebke I, Kampf G. How long do nosocomial pathogens persist on inanimate surfaces? A systematic review. BMC Infect Dis. 2006;6:130. doi:10.1186/1471-2334-6-130. 